\title{
A fuzzy inference approach to control robot speed in human-robot shared workspaces
}

\author{
Angelo Campomaggiore, Marco Costanzo, Gaetano Lettera and Ciro Natale \\ Dipartimento di Ingegneria, Università degli Studi della Campania "Luigi Vanvitelli”, Via Roma 29, Aversa, Italy \\ angelo.campomaggiore@studenti.unicampania.it,\{marco.costanzo,gaetano.lettera,ciro.natale\}@unicampania.it
}

\begin{abstract}
Keywords: Human-robot collaboration, Workspace monitoring, Fuzzy logic
Abstract: $\quad$ Nowadays, human-robot collaboration (HRC) is an important topic in the industrial sector. According to the current regulations, the robot no longer needs to be isolated in a work cell, but a collaborative workspace in which human operators and robots coexist can be acceptable. Human-robot interaction (HRI) is made possible by proper design of the robot and by using advanced sensors with high accuracy, which are adopted to monitor collaborative operations to ensure the human safety. Goal of this article is to implement a fuzzy inference system, based on the ISO/TS 15066, to correctly compute the minimum protective separation distance and adjust the robot speed by considering different possible situations, with the aim to avoid any collisions between operators and robots trying to minimize cycle time as well.
\end{abstract}

\section{INTRODUCTION}

The research paper tackles the human-robot collaboration problem by following the line of the current regulations and introducing a new approach to be used in manufacturing industry. The novel method assures human operators safety, without modifying the robot predefined path and defining a safety metric to scale robot trajectory only when indispensable, thus trying to maximize the production time.

The research work is carried out in the framework of a European project (The LABOR project, 2019), which has the objective to propose novel robotized assembly paradigms of aircraft fuselage panels. Until recently, the aerospace industry was still conservative and companies tended to use successful assembly methods that had already been proven to work in the past. Nowadays, many assembly sub-operations try to exploit robotics, e.g., drilling, fastening and sealing tasks. These operations are no longer manually performed by human operators but by industrial robots equipped with dedicated tools or by large automated machines dedicated to assembly of specific parts. However, there are some detailed operations

\footnotetext{
1(C2019 Scitepress. Presented @ICINCO 2019. DOI: http://dx.doi.org/10.5220/0007838700780087
}

which require human capabilities and that must be still executed by operators. This is the case of hybrid metal and composite structures, where, after the drilling operation, some parts have to be manually removed for further manual operations, like deburring, and then re-installed on the skin panel before the sealing and riveting operations, as shown in Figure 1 .

This requires to setup a robotic cell that has to foresee the presence of a human operator, hence the necessity to monitor the shared workspace. Real-time workspace monitoring for human-robot coexistence is not an easy problem to solve. Even more, implementing strategies to maximize the production time and preserve human safety at the same time is a research challenge. The approach proposed here is to adopt a fuzzy inference logic that can update the planned robot velocity in real-time according to robust perception data and a set of rules formulated based on a risk analysis. This can lead to a novel, acceptable solution.

Ensuring the safety of a human operator is the main purpose of the current research of industrial collaborative robotics. The safety standards for applications of industrial robots are laid out by the International Organization for Standardization (ISO) (ISO 10218-1, 2011), (ISO 10218-2, 2011), and by the upcoming ISO proposed draft Technical Specification (TS) (ISO/TS 15066, 2016), which addresses four collaborative scenarios:

1. Safety-rated Monitored Stop (SMS), which re- 
quires that the robot stops when a human is in the collaborative workspace;

2. Hand Guiding (HG), which allows the operator to hand-guide the robot through an hand guiding equipment (e.g., an analog button cell attached to the robot) and an emergency stop conforming to International Electrical Commission (IEC) (IEC) 60204-1, 2009);

3. Speed and Separation Monitoring (SSM), which monitors the robot speed according to the separation distance from the operator;

4. Power and Force Limiting (PFL), which limits the momentum of the robot such that the potential for operator injury upon impact is minimized, according to the established injury standards (Bicchi et al., 2008).

In this paper, a strategy to handle the operators safety in industrial SSM scenarios is investigated. The main goal is to reasonably scale down the size of the protective zone around the robot and improve productivity, taking into account safety regulations. The robot behavior is modified, in terms of trajectory scaling, only if there is a real and imminent risk of collision. The operator approach into the collaborative workspace is deeply analyzed to generalize the computing method of the safety index and face the extreme variability and unpredictability of human behaviours. The devised solution computes the points at minimum distance between the robot and the closest human and presents several desirable features with respect to other solutions, e.g., (Zhang et al., 2016),(Bascetta et al., 2011),(Lippi and Marino, 2018),(Bjerkeng et al., 2014); many of these approaches rely on evasive actions to increase safety. However, in industrial setting, it is generally recommended to follow the robot predefined path without deviating from it, especially in complex work cells, where clashes are likely to occur. The main characteristics of the proposed approach are:

- it considers the whole surface of human operators, without using skeleton-based techniques and without approximating the body to a single point;

- it considers the whole robot kinematic chain, the entire volume and possible tools, without factoring only a singular representative element of the robot (e.g., the end effector);

- it explicitly takes into account the regulations;

- it predicts the human velocity $v_{H}$, by estimating it from perception data without assuming it constant;

- it is based on a risk analysis that considers the relative directions of velocities, which are not taken

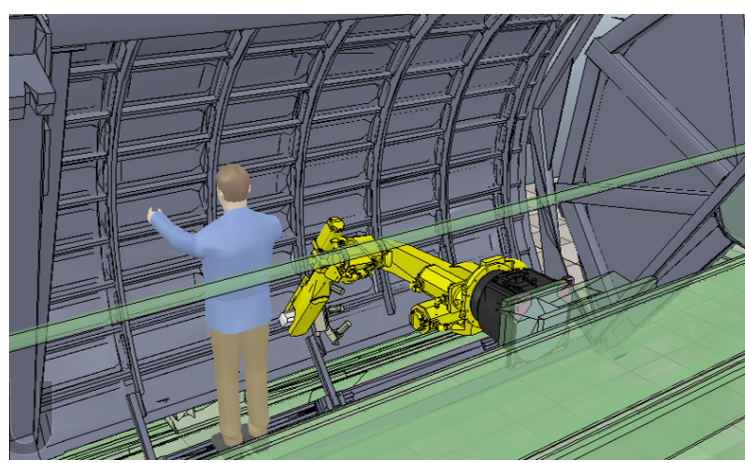

Figure 1: Example of a manual assembly operation where the operator shares the workspace with a robot.

into account in the equation proposed by the current regulations;

- it does not modify the robot programmed path and it does not require the task to be aborted.

\section{ISO ANALYSIS: SSM}

SSM allows the robot system and the operator to move concurrently in the collaborative workspace. Risk reduction is achieved by maintaining at least the minimum protective separation distance, $S$, between the human operator and the robot all the time. During robot motion, the robot system never gets closer to the operator than $S$. When the Euclidean separation distance, $d$, is equal to or less than $S$, the robot system stops, before it can impact the operator. When the operator moves away from the robot system, the robot system can resume the motion automatically while maintaining at least the protective separation distance.

(ISO 13855, 2010) is the first document which investigates the issue of safeguards positioning for human safety in stationary, active machinery. The document suggests to compute $S$ as

$$
S=v T+C,
$$

where $v$ is the approach speed of human body parts and its value is assumed to be as the maximum operator speed of $2.0 \mathrm{~m} / \mathrm{s}$, unless $d$ is greater than $0.5 \mathrm{~m}$, in which case may be set at $1.6 \mathrm{~m} / \mathrm{s}$. $T$ is the total system stopping performance time, in seconds, and it is a combination of the time required by the machine to respond to the operator's presence (i.e., $T_{R}$ ) and the response time of the machine which brings the robot to a safe, controlled stop (i.e., $T_{S}$ ). $C$ is the intrusion distance safety margin, which represents an additional distance, based on the expected intrusion toward the critical zone prior to the actuation of the protective equipment. 
From eq. (1), ISO/TS 15066 updates the $S$ meaning by including robot dynamic properties. When the robot system reduces its speed, the protective separation distance decreases correspondingly, i.e.,

$$
\begin{aligned}
S\left(t_{0}\right) \geq & \int_{\tau=t_{0}}^{\tau=t_{0}+T_{R}+T_{S}} v_{H}(\tau) d \tau+\int_{\tau=t_{0}}^{\tau=t_{0}+T_{R}} v_{R}(\tau) d \tau \\
& +\int_{\tau=t_{0}+T_{R}}^{\tau=t_{0}+T_{R}+T_{S}} v_{S}(\tau) d \tau+\left(C+Z_{S}+Z_{R}\right)
\end{aligned}
$$

In (2), $v_{H}$ is the "directed speed" of the closest operator which travels toward the robot, $v_{R}$ is the speed of the robot in the direction of the operator, $v_{S}$ is the directed speed of the robot in course of stopping. The remaining terms represents uncertainties: the intrusion distance $C$ is based on the operator reach, $Z_{R}$ is the robot position uncertainty, and $Z_{S}$ is the operator position uncertainty (i.e., the sensor uncertainty). Finally, $t_{0}$ is considered the current time.

The main issue of (ISO 13855, 2010) is that the separation distance was initially intended for static machinery, not for dynamic and reconfigurable robotic systems. Therefore, extending what is contained in the standard to the case of industrial robotics is not trivial. Nevertheless, ISO/TS 15066 tries to make a contribution to the HRC problem and describes $S$ using the linear function

$$
S=\left(v_{H} T_{R}+v_{H} T_{S}\right)+\left(v_{R} T_{R}\right)+(B)+\left(C+Z_{S}+Z_{R}\right)
$$

where $B$ is the Euclidean distance travelled by the robot while braking. Note the one-to-one correlation between eq. (2) and the linear relationship (3). The first term in parentheses describes the contribution attributable to the operator's change in location in the time necessary to bring the robot to a full stop from its current speed. The second term describes the contribution attributable to the robot system reaction time, before it initiates the braking sequence. The third term describes the distance travelled by the robot during its braking. Finally, the fourth term describes the possible distance of intrusion into the robot work volume as a function of the operator reach and the uncertainty of the sensory system and robot kinematics. The values of $v_{H}, T_{S}, B$ and $C$ can be found in the safety standards: the values of $v_{H}$ and $C$ are given in ISO 13855 , while guidelines for evaluating $T_{S}$ and $B$ are given in Annex B of ISO 10218-1 and they result from measurements that directly depend on the robot system under test.

This paper decomposes and assesses the performance of ISO/TS 15066 SSM minimum protective distance metric and adds a contribution to improve some aspects to allow its applicability in industrial scenarios. The following sections widely discuss four main areas that are directly pertinent to SSM: human detection and tracking, prediction of human and robot motions, safety separation maintenance and robot speed monitoring.

\section{HUMAN-ROBOT INTERACTION}

The robot control system must be able to adapt the robot trajectory to the current observed scene and to perform its task efficiently and safely. This means that the control system must be able to detect the presence of human operators inside the collaborative workspace, to track the human closest to the machine and, finally, to modulate the robot speed according to the minimum protective distance $S$.

The HRC has been addressed dividing it into two distinct problems: human detection and tracking (HDT) and intention estimation (IE).

\subsection{Perception System}

The experimental set-up of this work is composed by two depth cameras, which have been used to monitor the collaborative workspace: a Microsoft Kinect $v 1$ and an Intel RealSense D435 (see Figure 2a). At least two views become necessary to minimize the occlusions of the observed area, as shown in Figure $2 b$ and Figure 2c

An intrinsic calibration is necessary to update the rough intrinsic default parameters, as well as, a sphere-tracking procedure has been developed for extrinsic calibration. The obtained homogeneous transformation matrices, $T_{\text {camera } 1}^{\text {robot }}$ and $T_{\text {camera } 2}^{\text {robot }}$, express the poses of the camera frames with respect to the robot base frame.

The goal of the extrinsic calibration is to obtain an accurate identification of the camera pose, which guarantees the minimum relative positioning error when the two camera views are merged.

Therefore, a 3D tracking technique has been developed by using a polystyrene sphere of $0.12 \mathrm{~m}$ diameter. The red sphere has been mounted at the robot end effector, so as to match the center of the sphere with the origin of the end-effector frame, as shown in Figure 2. The calibration procedure uses the M-estimator SAmple Consensus (MSAC) algorithm (Torr and Murray, 1997) (which is an extension of the best known RANdom SAmple Consensus (RANSAC) algorithm (Fischler and Bolles, 1981)), to find a sphere within a radius constraint, and to provide its geometric model. The robot has been positioned at specific configurations, which allow to correctly 


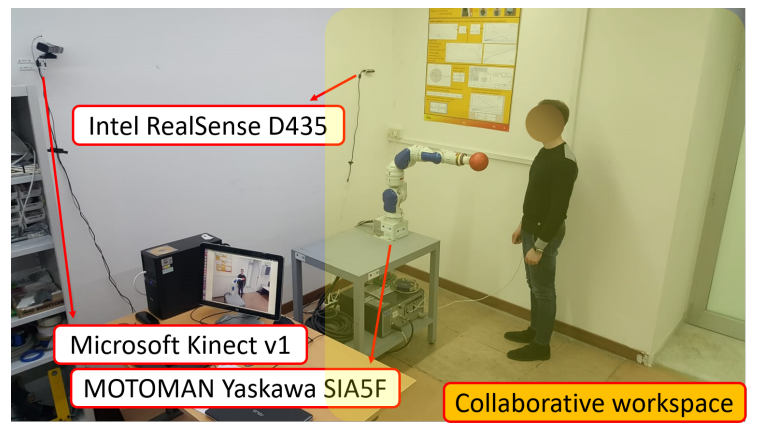

(a) Perception system.

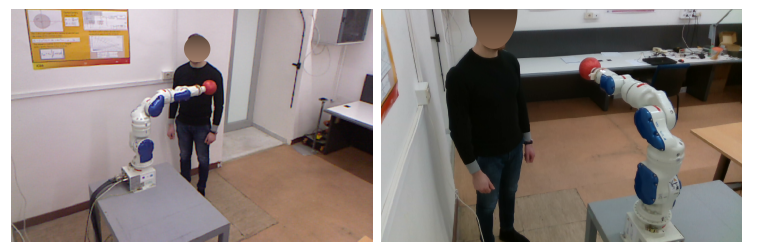

(b) Kinect RGB view.

(c) RealSense RGB view.

Figure 2: Experimental set-up.

distinguish the target within the two camera views. From the robot joint states, the forward kinematics computes the pose of the center of the red sphere. At the same time, the developed procedure acquires the depth images, converts them into point clouds (Rusu and Cousins, 2011) and estimates the target model. The method is iterated to cover the entire collaborative workspace and to minimize the positioning error. Finally, the transformation matrices have been evaluated through an optimization algorithm with a cost function that combines the data of both cameras.

\subsection{Human Detection and Tracking}

Realizing a safe HRC application requires a very fast HDT algorithm, which detects human operators in real time. In this study, a novel point cloud-based methodology is presented to compute the minimum distance between the whole body of the detected operators and a robot. Since this operation is computationally heavy, a Background Segmentation (BS) algorithm is developed to subtract the static environment from the observed scene and to process exclusively the information related to the dynamic objects. The developed pipeline is shown in Figure 3 .

The perception system described in Section 3.1 observes the surroundings of the manipulator and the robot kinematic chain is fully visible. While the workspace is monitored, the robot executes its task, thus it becomes a dynamic entity. Therefore, the $R e$ altime URDF Filter (Blodow, 2012) is used to remove the robot from the scene.

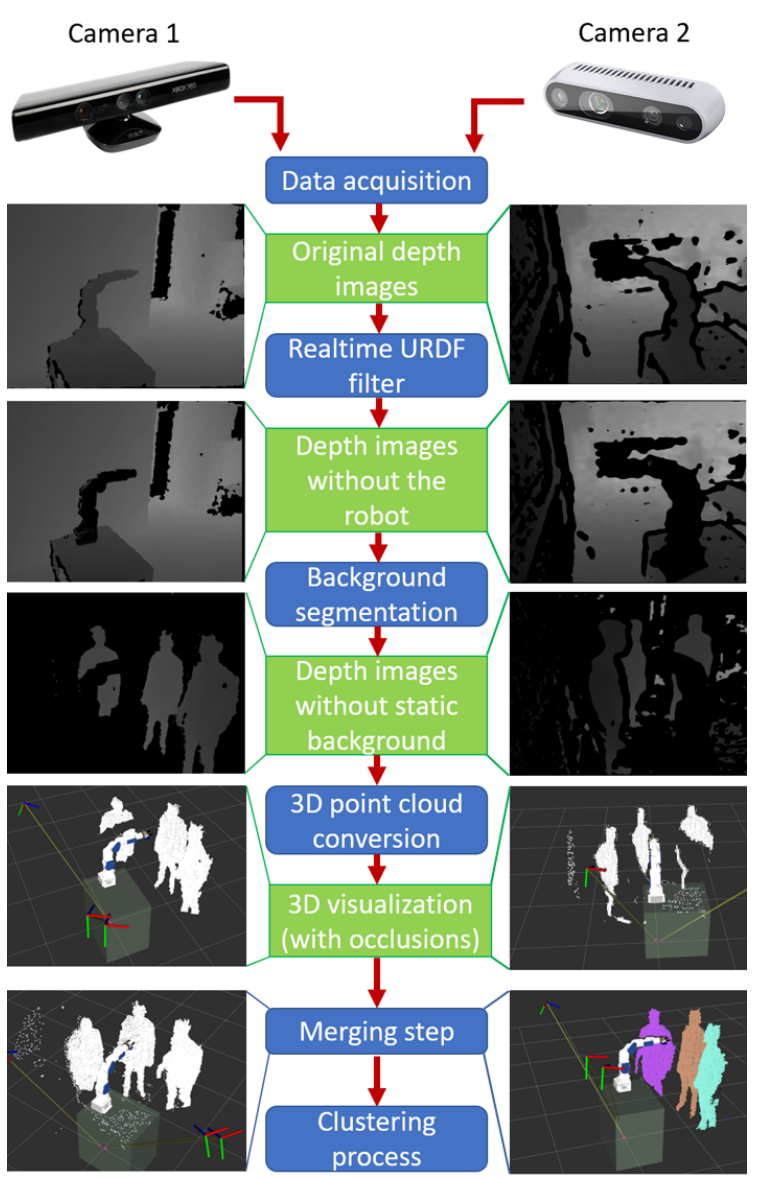

Figure 3: Implemented HDT pipeline.

The implementation of the BS step consists of an efficient algorithm that performs the subtraction of a stored background, at pixel level: 50 frames of a static scene in the absence of human workers are initially captured and the mean value of each pixel is stored in a memory area. Therefore, the stored frame is subtracted from the current frame at every acquisition.

The algorithm makes use of PCL: the depth information is converted into Point Cloud Data (PCD) and a uniform sampling filter can be applied to make the algorithm more reactive, by decreasing the PCDs density.

Subsequently, a reference camera has been selected to express the entire output of the perception system relative to a single camera frame, in this case, the Kinect camera. The point clouds have been combined through the merging step (MS). The accuracy reached during the extrinsic calibration procedure, described in Section 3.1, allowed to obtain a satisfying correspondence.

Finally, the clustering process $(\mathrm{CP})$ provides as many clusters as single dynamic areas are detected 
in the foreground. The Euclidean cluster extraction method is performed to highlight all the human clusters of the collaborative workspace. The bottom right image of Figure 3 shows three detected human operators, whose shapes are distinguishable by different colors. To compensate the sensors measurement noise that could sometimes provide false clusters, the areas in the foreground should be large enough to represent a human body. Therefore, a valid cluster should have a minimum PCD cardinality, empirically determined.

\subsection{Human-Robot Separation Distance}

The goal of the proposed HRC strategy is to identify the nearest pair of points, one belonging to the robot $\left(P_{R}\right)$ and the other one belonging to the operator $\left(P_{H}\right)$, that minimize the distance, i.e.,

$$
\begin{array}{r}
P_{H} \in \mathcal{H}, P_{R} \in \mathcal{R} \mid d\left(P_{H}, P_{R}\right) \leq d\left(P_{H}^{\prime}, P_{R}^{\prime}\right) \\
\forall P_{H}^{\prime} \in \mathcal{H}, P_{R}^{\prime} \in \mathcal{R}
\end{array}
$$

where $d(\cdot, \cdot)$ is the Euclidean distance between two points, $\mathcal{H}$ and $\mathcal{R}$ represent the set of all points that belong to the operator and to the robot, respectively.

Therefore, alongside the HDT strategy, a robot modeling method has been also implemented. To the best of authors knowledge, the typical SoA assumption is to consider only a representative elements of the robot (e.g., the end effector), introducing only an approximate estimation of the distance between the operators and the robot kinematic chain. Other solutions report the pose of the robot only in terms of either joint configurations or in terms of the Cartesian pose of the robot link frames, without taking into account the link shapes but considering only specific points. On the contrary, the proposed solution models the entire robot kinematic chain with its volume. A computationally efficient way to represent the whole robot is to use primitive shapes, e.g., ellipses and spheres (Choi and Kim, 1999). A similar convention was proposed in (Bosscher and Hedman, 2009) . This work is inspired by the same idea, but pays attention to some aspects: since the robot links can have different lengths, its kinematic chain has been padded through dummy frames to protect the robot homogeneously, and a $0.10 \mathrm{~m}$ diameter security sphere has been created around each frame, taking into account the last frame that can incorporate an end-effector tool.

Under such assumptions, the pair of human-robot points that are closest to each other can be immediately identified. This step strongly justifies the choice of a point cloud-based pipeline. In fact, the point cloud provides much more detailed information, accuracy and precision if compared to the major HDT

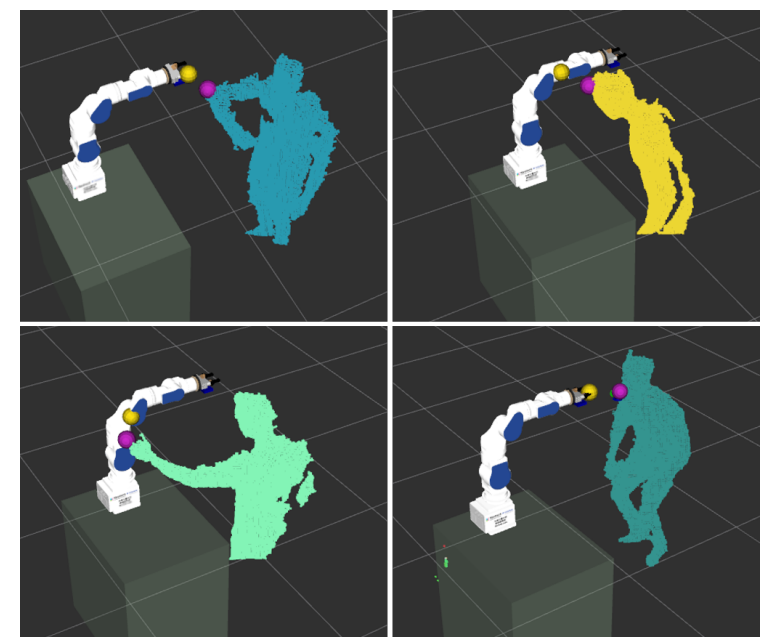

Figure 4: Identification of the minimum distance points: the yellow sphere is the robot point closest to the human and the purple one is the human point closest to the robot.

techniques present in the SoA literature cited in Section 1. Unlike common skeleton-based techniques, the proposed approach allows tracking humans also when they are carrying objects. Moreover, it is not necessary that human operators are in front of the camera view: the point cloud will recognize them anyway. Furthermore, detecting the pair of humanrobot points at minimum distance (4) is particularly immediate. The algorithm calculates the distance between all points of a cluster point cloud and the origin of every robot frame. Eventually, the robot point $P_{R}$ will be the one on the surface of the virtual sphere, around the identified frame, which lies on the line connecting the origin of this frame and the closest point in the cluster. From these results, the closest human cluster is indirectly selected if more than one human have been detected.

Figure 4 shows the results. Note that the proposed approach is able to identify more detailed body parts, e.g., a elbow, the head, an hand, the chin or the chest, and also that $P_{R}$ can be detected along the whole robot kinematic chain. Figure 5 demonstrates the effectiveness of the proposed approach in multi-humans scenarios. The results of the experimental tests described in Section 6 will be used to evaluate the performances of the algorithm.

\subsection{Estimation of Operator and Robot Velocities}

Another fundamental function of the HRC problem is represented by IE, i.e., the prediction of human movement. From such information, the robot control system will select the most appropriate value of its joint 

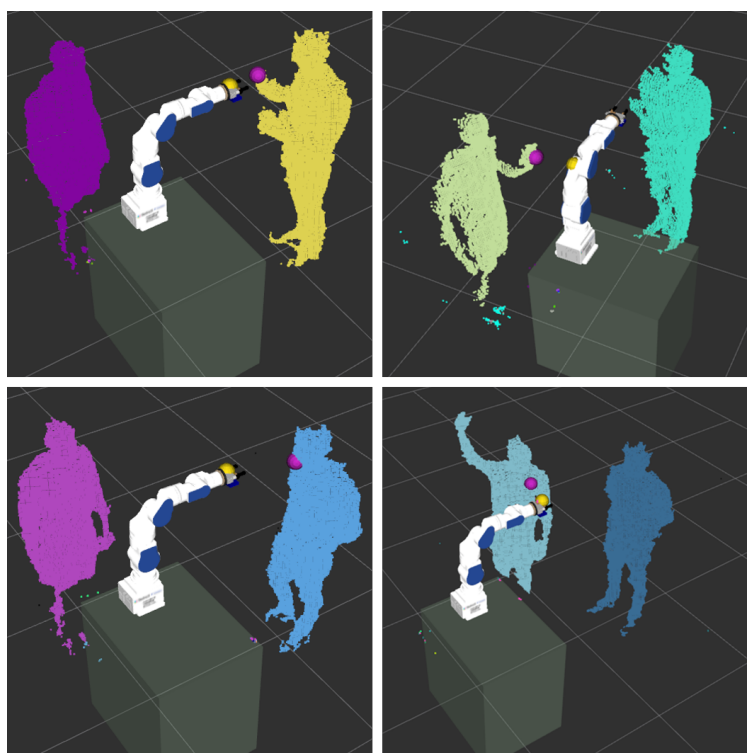

Figure 5: Multi-humans tracking.

speeds to avoid a potentially dangerous situation, as explained in Section 5 .

IE consists in estimating the next position and velocity of the trajectory performed by the operator on the basis of a series of positions previously acquired.

The sensor fusion strategy that has been integrated into this work is based on a Linear Kalman Filter (LKF), which tries to solve the problem of estimating the state of a discrete-time process governed by the equations

$$
\begin{aligned}
\boldsymbol{x}_{k+1} & =\left[\begin{array}{cc}
\boldsymbol{I}_{3} & \Delta t \boldsymbol{I}_{3} \\
\boldsymbol{O}_{3} & \boldsymbol{I}_{3}
\end{array}\right] \boldsymbol{x}_{k}+\boldsymbol{w}_{k}, \\
\boldsymbol{y}_{k} & =\left[\begin{array}{ll}
\boldsymbol{I}_{3} & \boldsymbol{O}_{3}
\end{array}\right] \boldsymbol{x}_{k}+\boldsymbol{n}_{k}
\end{aligned}
$$

where $\Delta t$ is the sampling time, $\boldsymbol{I}_{3}$ and $\boldsymbol{O}_{3}$ are the identity and zero matrices of size $3 \times 3$, respectively; $\boldsymbol{w}$ and $\boldsymbol{n}$ are the process and measurement noises with covariance matrices $\boldsymbol{W}$ and $\boldsymbol{N}$, respectively. Finally, $\boldsymbol{x}$ is the state vector of the system, i.e., the position and the velocity of the operator $\boldsymbol{x}=\left[\begin{array}{ll}\boldsymbol{p}_{H}^{T} & \dot{\boldsymbol{p}}_{H}^{T}\end{array}\right]^{T}$, and the measured output $\boldsymbol{y}$ is a vector containing the coordinates of the point $P_{H}$ described in Section 3.3 . The covariance matrix $N$ is experimentally estimated, while the covariance matrix $Q$ has been chosen as

$$
\boldsymbol{Q}=\left[\begin{array}{cc}
\boldsymbol{I}_{3} \Delta t^{2} & \boldsymbol{O}_{3} \\
\boldsymbol{O}_{3} & Q_{2}
\end{array}\right]
$$

where $Q_{2}$ quantifies the uncertainty on the velocity dynamics (assumed constant) of the state equations.

Based on the vector nature of the velocity, it is possible to make some considerations about the direction (trend) of the operator, that is to say, to predict in which direction he/she is travelling to. Section 4
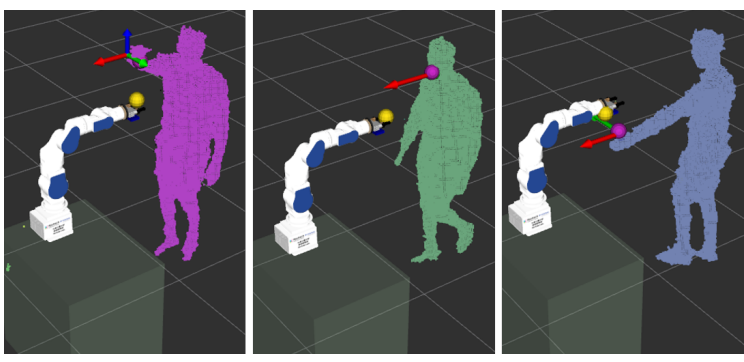

Figure 6: Estimation of operator velocity.

describes how to take advantage from these considerations for industrial collaborative applications with the aim to maximize productivity.

The LKF equations implemented in this work are the standard ones and thus are not reported for brevity, while the tuned parameters are fully described in Section 6 .

Figure 6 shows sample movements of the operator and the three components of his/her estimated speed.

The linear velocity $\dot{p}_{R}$ of the point on the robot closest to the operator can be computed according to the differential kinematics equation

$$
\dot{\boldsymbol{p}}_{R}=\boldsymbol{J}_{p}(\boldsymbol{q}) \dot{\boldsymbol{q}}
$$

where $\boldsymbol{q}[\mathrm{rad}]$ and $\dot{\boldsymbol{q}}[\mathrm{rad} / \mathrm{s}]$ are the robot joint position and velocity vectors, respectively; while, $\boldsymbol{J}_{p}$ is the position part of the Jacobian matrix calculated till the closest point.

The (ISO/TS 15066, 2016) states that the "directed speeds" of the robot and the human should be used to compute $S$. This means that, in eq. (3), $v_{h}$ is the operator speed in the direction of the moving part of the robot and $v_{R}$ is the robot speed in the direction of the selected operator. Note also that these speeds are vector magnitudes, hence they are always grater or equal to 0 . Therefore, the velocity terms of (3) can be computed as

$$
\begin{gathered}
v_{H}=\left|\dot{\hat{\boldsymbol{p}}}_{H}^{T}\left(\frac{\boldsymbol{p}_{R}-\hat{\boldsymbol{p}}_{H}}{\left\|\boldsymbol{p}_{R}-\hat{\boldsymbol{p}}_{H}\right\|}\right)\right| \\
v_{R}=\left|\dot{\boldsymbol{p}}_{R}^{T}\left(\frac{\hat{\boldsymbol{p}}_{H}-\boldsymbol{p}_{R}}{\left\|\hat{\boldsymbol{p}}_{H}-\boldsymbol{p}_{R}\right\|}\right)\right|,
\end{gathered}
$$

where $\hat{\boldsymbol{p}}_{H}$ and $\dot{\hat{\boldsymbol{p}}}_{H}$ are the operator position and velocity estimated by the LKF, respectively, and $p_{R}$ is a vector containing the coordinates of the point $P_{R}$ defined in Section 3.3

\section{FUZZY INFERENCE SYSTEM}

The protective separation distance $S$ in (3), computed by using the speeds of $(9)-(10)$, does not take into account the relative travel direction of the robot and the 
operator. This means that, if the robot and the operator are going away from each other, the value of $S$ unnecessarily increases (proportionally to the computed speed). To improve the production time considering also this situation, the protective separation distance has been redefined as follows

$S=\alpha\left[\left(v_{H} T_{R}+v_{H} T_{S}\right)+\left(v_{R} T_{R}\right)\right]+(B)+\left(C+Z_{S}+Z_{R}\right)$,

where $\alpha$ is a coefficient in the interval $[0,1]$ that is 1 when the operator and the robot are actually approaching to each other and is smaller than 1 otherwise.

To chose the value of $\alpha$, a fuzzy inference approach has been implemented. The fuzzy logic, also called faded logic, is a methodology in which each proposition possesses a degree of truth into the interval $[0,1]$ (Ross, 2010). The variable $\alpha$ must be classified taking into account some qualitative attributes and it may have varying levels of validity between a maximum (1) and a minimum (0). Hence, it is necessary to generate linguistic rules of fuzzy inference to realize a mapping of the inputs to the desired output.

The fuzzy inference process has been developed as a two-input, one-output, three-rule problem, as shown in Figure 7 .

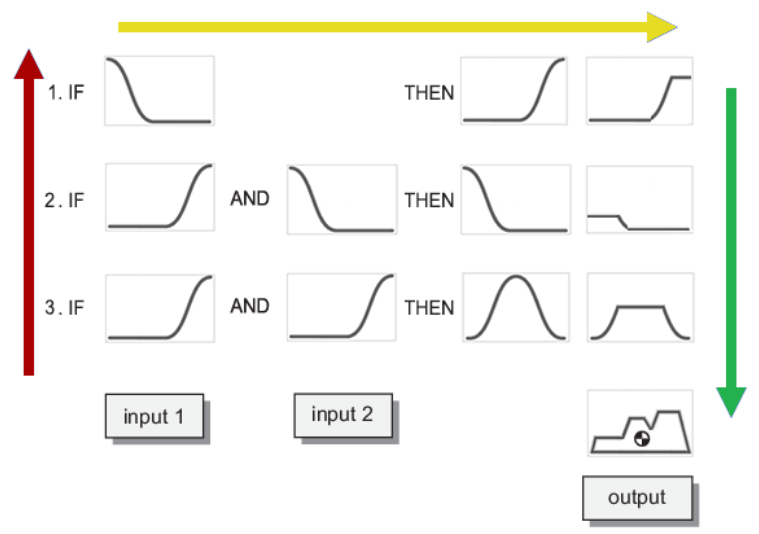

Figure 7: Fuzzy inference system: the fuzzification step (red arrow), the implication step (yellow arrow) and the aggregation step (green arrow).

The first step is to select the inputs. Two data inputs have been selected:

1. the time derivative of the distance between human and robot, i.e., $\dot{d}=\frac{\mathrm{d}\left\|\hat{\boldsymbol{p}}_{H}-\boldsymbol{p}_{R}\right\|}{\mathrm{d} t}$;

2. the scalar product between the robot and the human velocity vectors, i.e., $\dot{\boldsymbol{p}}_{R}^{T} \dot{\hat{\boldsymbol{p}}}_{H}$.

The first input is useful to distinguish cases when the operator and the robot are getting closer and cases when they are moving away from each other. The scalar product specifies the relative direction of travel of the operator and the robot.

The next step is the fuzzification step (red arrow of Figure 7). The ranges of variability of each input have been defined, and the appropriate membership function of each interval has been selected. This step requires attention to correctly determine the degree to which the input belongs to each of the appropriate fuzzy set, by assigning a fuzzy degree of membership in the interval from 0 to 1 . Two membership functions have been selected to represent positive $(\mathrm{P})$ and negative $(\mathrm{N})$ values, a $\mathrm{Z}$-shape and a $\mathrm{S}$-shape, respectively. These functions, with different parameters, have been chosen to describe both $\dot{\boldsymbol{p}}_{R}^{T} \dot{\hat{\boldsymbol{p}}}_{H}$ and $\dot{d}$.

After the inputs are fuzzified, the implication step (yellow arrow of Figure 7) determines the degree to which each part of the antecedent is satisfied for each rule. The antecedent of the developed fuzzy inference rules has three parts, combined through an AND method ( $\mathrm{min}$ ) to obtain an implicated number that represents the result of the rule antecedent. Each rule is designed to consider one possible risk scenario.

Since the final decision is based on the result of all the tested rules, the outputs of the rules must be combined in some way. The aggregation step (green arrow of Figure 7) is the process by which the fuzzy sets representing the outputs of each rule are combined into a single fuzzy set, before the last defuzzification step. For each interval of the consequent, the maximum value of the fuzzy set is chosen and the defuzzification method is the centroid, as shown at the end of Figure 7 .

The output value, $\alpha$, has been generated by analyzing different possible risk situations, with the twofold aim of avoiding any collisions between human and robot, and being in line with the current ISO/TS 15066. With reference to the second rule: if the human-robot distance is increasing and they are moving further from each other, than the safety distance can be decreased. The three rules are summarized in Table 1 .

Table 1: Fuzzy rules: [S] Small, [M] Medium, [H] High, $[\mathrm{N}]$ Negative, [P] Positive, $[\sim]$ any.

\begin{tabular}{|c|c|c|}
\hline \multicolumn{2}{|c|}{ antecedent } & consequent \\
\hline$\dot{d}$ & $\dot{\boldsymbol{p}}_{R}^{T} \dot{\hat{\boldsymbol{p}}}_{H}$ & $\alpha$ \\
\hline $\mathrm{N}$ & $\sim$ & $\mathrm{H}$ \\
$\mathrm{P}$ & $\mathrm{N}$ & $\mathrm{S}$ \\
$\mathrm{P}$ & $\mathrm{P}$ & $\mathrm{M}$ \\
\hline
\end{tabular}

Note that the scalar product between the operator velocity and the robot velocity (second input) is a complementary information to the time derivative of the distance between human and robot (third input). Since $\dot{\boldsymbol{p}}_{R}^{T} \dot{\hat{\boldsymbol{p}}}_{H}=\left\|\dot{\boldsymbol{p}}_{R}\right\|\left\|\dot{\hat{\boldsymbol{p}}}_{H}\right\| \cos \theta$, when $\theta=180^{\circ}$, 

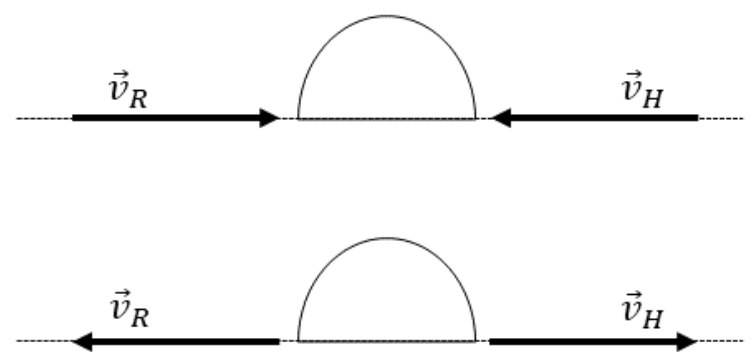

Figure 8: Problem of the scalar product.

a critical situation is possible. The result of the scalar product is negative, $\dot{\boldsymbol{p}}_{R}^{T} \dot{\hat{\boldsymbol{p}}}_{H}<0$, but it is not possible to distinguish the cases shown in Figure 8 in which the directions are opposite but it is not known if the human and the robot are getting closer or are moving away from each other. This is the reason why it is necessary to combine the scalar product information with the time derivative of the distance between the human operator and the robot.

\section{TRAJECTORY SCALING}

SSM scenarios usually sacrifice the production time because a lot of time is spent in low speed mode when a human operator is inside the collaborative workspace. On the contrary, the proposed strategy ensures human-robot coexistence according to the standard regulations, and also guarantees the task efficiency by using a time-scaling approach to change robot operating speed without introducing acceleration discontinuities.

A typical industrial pre-programmed task, $\mathcal{T}$, is composed by $N$ positions $\tilde{q}_{i}$, associated to velocities $\dot{\tilde{q}}_{i}$, accelerations $\ddot{\tilde{q}}_{i}$ and time instants $\tilde{t}_{i}$ with $i=$ $1, \ldots, N$. Typically, the pre-programmed joint positions have to be interpolated according to the sampling time $T_{c}$ required by the robot control interface. In this work a quintic interpolation is used, i.e., the planned interpolated trajectory is

$$
\begin{gathered}
\tilde{q}_{h}=p_{5}\left(t_{h} ; \mathcal{T}\right) \\
\dot{\tilde{q}}_{h}=p_{4}\left(t_{h} ; \mathcal{T}\right) \\
t_{h+1}=t_{h}+T_{c},
\end{gathered}
$$

where $t_{h}$ is the $h$-th discrete time instant, $p_{4}$ is the derivative of the polynomial $p_{5}, \tilde{q}_{h}$ and $\dot{\tilde{q}}_{h}$ are the planned joint position and velocity at time $t_{h}$, respectively.

The proposed method modulates the robot speed by scaling the time with a safety scale factor $k$, which can assume values in the interval $[0,1]$. The scale factor is related to $d$ (Section 3.3) as shown in Figure 9

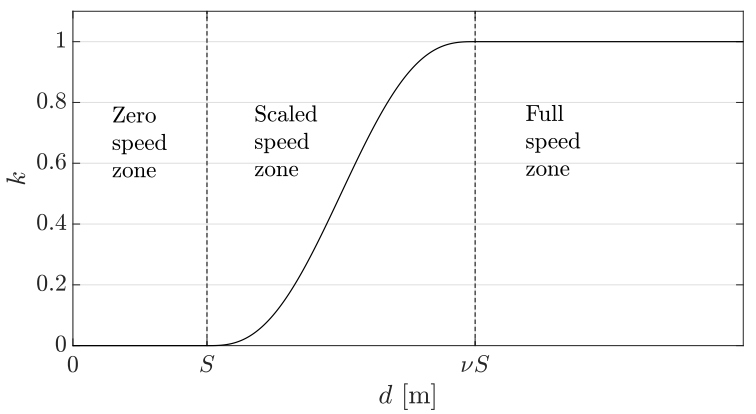

Figure 9: Relation between $d$ and $k$.

When $d$ is below the minimum protective distance $S$, $k$ is 0 and the robot stops. When the distance $d$ is far from $S$, i.e. $d>v S(v>1)$, the robot can move at full speed to improve the production time. Between $S$ and $\mathrm{v} S$ the function in Figure 9 smoothly varies to avoid acceleration discontinuities. Obviously, $v$ is another design parameter that changes the size of the scaled speed mode zone.

Practically, the trajectory is scaled computing 12 using a scaled time $\tau_{h}$, i.e.,

$$
q_{h}=p_{5}\left(\tau_{h} ; \mathcal{T}\right) \quad \tau_{h+1}=\tau_{h}+k T_{c},
$$

where $q_{h}$ is the actual joint command at time $t_{h}$. Obviously, the joint command $q_{h}$, as well as the scaled time $\tau_{h}$, are generated with sampling time $T_{c}$.

This approach effectively scales the joints velocities. In fact, using (15), it is

$$
\dot{\tau} \approx \frac{\tau_{h+1}-\tau_{h}}{T_{s}}=k .
$$

By time differentiating (15), (17) demonstrates that the velocity is scaled by the safety factor $k$,

$$
\dot{q}_{h}=p_{4}\left(\tau_{h} ; \mathcal{T}\right) k . .
$$

This approach guarantees that the task $\mathcal{T}$ remains the same in position, but, simultaneously, the resulting velocity is scaled according to $k$.

When the operator is going to be into a dangerous situation, the robot operates at diminished capacity with limits on velocity that respect human-robot collaboration norms, until restoration of the safety conditions. Note that the side effect of the velocity reduction is the reduction of the minimum protective distance $S$, since this value is proportional to the robot velocity. Experimental results are shown in Section 6

\section{EXPERIMENTAL RESULTS AND VALIDATION}

This section shows an example of experimental results obtained by simulating an SSM human-robot 
collaboration task inside the collaborative workspace of Figure 2. A manufacturing industrial sealing operation has been virtually realized: the robot executes a pre-planned path at a given nominal speed, while, suddenly, a human operator enters the collaborative workspace to perform some manual operation close to the robot, at different distances.

The main goal of this experiment is to prove the efficiency of the fuzzy inference approach into industrial applications to better handle the production time and, at the same time, to guarantee the safety of the operators when they are inside the collaborative workspace.

Table 2 summarizes the used hardware and the experimental case study.

Table 2: Case study and available hardware.

\begin{tabular}{|c|c|}
\hline Robot & Yaskawa SIA5F \\
\hline Collaborative workspace & $4 \times 2 \mathrm{~m}$ \\
\hline Depth camera (1) & Microsoft Kinect v1 \\
\hline Depth camera (2) & Intel RealSense D435 \\
\hline Robot simulated task & Sealing operation \\
\hline Operator simulated task & Manual piece change \\
\hline
\end{tabular}
as

The covariance matrix $\boldsymbol{Q}_{\mathbf{2}}$ in (7) has been chosen

$$
\boldsymbol{Q}_{2}=\operatorname{diag}(0.02,0.05,0.05) \mathrm{m}^{2} / \mathrm{s}^{2},
$$

while the noise covariance has been estimated by acquiring a constant human position as

$$
\boldsymbol{N}=\operatorname{diag}(0.0009,0.0008,0.001) \mathrm{m}^{2} \text {. }
$$

The parameters to compute the protective separation distance $S$ of (3) and (11) are reported in Table 3 . The value of $C$ has been chosen to better appreciate the zero speed zone.

Table 3: Constant parameters of $S$.

\begin{tabular}{|c|c|}
\hline$T_{R}$ & $0.10 \mathrm{~s}$ \\
\hline$T_{S}$ & $0.08 \mathrm{~s}$ \\
\hline$B$ & $0.563 \mathrm{~mm}$ \\
\hline$Z_{R}$ & $0.001 \mathrm{~m}$ \\
\hline$Z_{S}$ & $0.1067 \mathrm{~m}$ \\
\hline$C$ & $0.20 \mathrm{~m}$ \\
\hline
\end{tabular}

Figure 10 shows the results of the experiment. The graph at the top of the figure shows the distance between the human operator and the robot and it can be compared with the minimum protective distance computed as in 3 ( $S_{I S O}$ in the legend) and the two thresholds proposed in this paper: $S$ in the legend is the protective distance computed as in (11) and $v S$ is the threshold used in the trajectory scaling algorithm (Section 5). The bottom plot of Figure 10 shows the two inputs of the fuzzy inference system ( $\dot{d}$ and
$\left.\dot{\boldsymbol{p}}_{R}^{T} \dot{\hat{\boldsymbol{p}}}_{H}\right)$ and the trajectory scale factor $k$. In this experiment $S_{I S O}$ is not used and it is showed in the plot for comparison purposes. A video of the experiment is available at https: //youtu.be/RzLZ6RQBPCY

The robot executes a planned task, suddenly (at about $16 \mathrm{~s}$ ) an operator enters into the workspace simulating a manual task. This is visible in the top plot of Figure 10, where the human-robot distance decreases. Note that for almost the whole task duration the separation distance robot-operator is below the $S_{I S O}$ signal, this would have caused frequent starts and stops of the robot. Instead, through the proposed trajectory scaling algorithm, the robot reduces its velocity according to the observed separation distance. This is visible in the $k$ signal of the bottom plot that varies according to $d$. Notice that $k$ goes to 0 only when the distance $d$ goes below the protective distance $S$. Moreover, another property of the proposed solution is that $S$ increases only when the distance decreases (i.e., when $\dot{d}<0$ ) and not when the distance increases. This is due to the computation of the directed speed and the fuzzy rules. The shown experiment and the related video demonstrate how the proposed approach guarantees a safe human-robot coexistence in the collaborative workspace. This is achieved both in accordance with the ISO/TS regulations and minimizing dead times in the production process.

\section{CONCLUSIONS}

The human-robot interaction and their intentions to compete or cooperate in collaborative workspaces are challenging research fields. The purpose of this work is to improve the current regulations both to maximize the production time and guarantee the safety of human operators inside the shared workspace. The expected human movements relative to the robot are classified to identify all possible industrial SSM scenarios from which fuzzy rules for the robot reactions are derived. Collisions between robot and human operators are avoided by identifying human-robot intersections through a detection algorithm which processes data obtained by merging two depth camera images. Results obtained from experimental data show the applicability of the presented methods to many common manufacturing industry applications.

\section{ACKNOWLEDGEMENTS}

This work has received funding from the Clean Sky Horizon 2020 Programme under the LABOR project, grant agreement n. 785419. 

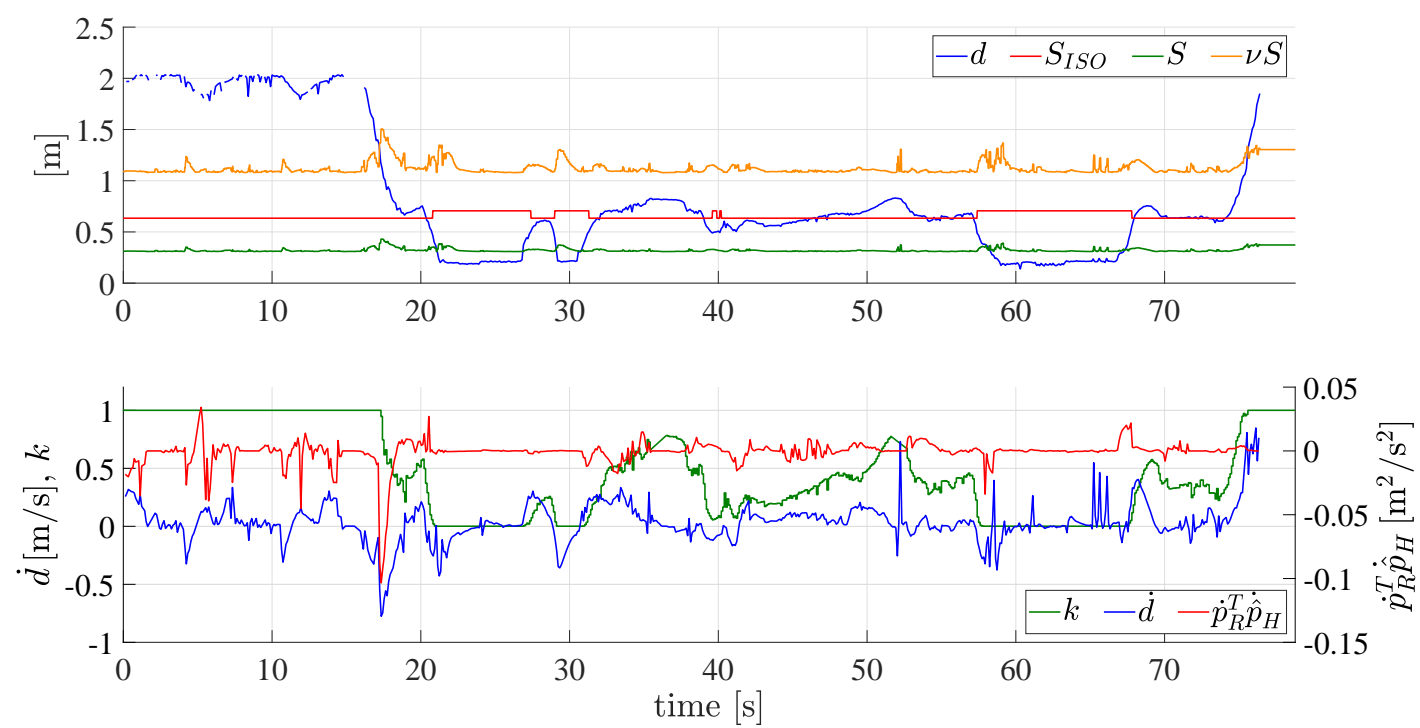

Figure 10: Experiment: an operator enters the shared workspace while the robot is moving. The top plot shows the estimated distance robot-operator $(d)$, the protective distances proposed by the regulation without sensing $\left(S_{I S O}\right)$ and the protective distances proposed by the paper $(S$ and $v S$ ). The bottom plot shows the trajectory scaling factor $k$, the time derivative of the distance $d$ and the scalar product of velocities.

\section{REFERENCES}

Bascetta, L., Ferretti, G., Rocco, P., Ardo, H., Bruyninckx, H., Demeester, E., and Lello, E. D. (2011). Towards safe human-robot interaction in robotic cells: An approach based on visual tracking and intention estimation. In 2011 IEEE/RSJ International Conference on Intelligent Robots and Systems. IEEE.

Bicchi, A., Peshkin, M. A., and Colgate, J. E. (2008). Safety for physical human-robot interaction. In Springer Handbook of Robotics, pages 1335-1348. Springer Berlin Heidelberg

Bjerkeng, M., Falco, P., Natale, C., and Pettersen, K. Y. (2014). Stability analysis of a hierarchical architecture for discrete-time sensor-based control of robotic systems. IEEE Transactions on Robotics, 30(3):745753.

Blodow, N. (2012). Realtime urdf filter.

Bosscher, P. and Hedman, D. (2009). Real-time collision avoidance algorithm for robotic manipulators. In 2009 IEEE International Conference on Technologies for Practical Robot Applications. IEEE.

Choi, S. I. and Kim, B. K. (1999). Obstacle avoidance control for redundant manipulators using collidability measure. In Proceedings 1999 IEEE/RSJ International Conference on Intelligent Robots and Systems. Human and Environment Friendly Robots with High Intelligence and Emotional Quotients (Cat. No.99CH36289). IEEE.

Fischler, M. A. and Bolles, R. C. (1981). Random sample consensus: a paradigm for model fitting with applications to image analysis and automated cartography. Communications of the ACM, 24(6):381-395.

IEC 60204-1 (2009). Safety of machinery - Electrical equipment of machines - Part 1: General require- ments. Technical report, International Electrotechnical Commission.

ISO 10218-1 (2011). Robots and robotic devices - Safety requirements for industrial robots. Part 1: Robots. Technical report, International Organization for Standardization.

ISO 10218-2 (2011). Robots and robotic devices - Safety requirements for industrial robots. Part 2: Robot system and integration. Technical report, International Organization for Standardization.

ISO 13855 (2010). Safety of machinery - Positioning of safeguards with respect to the approach speeds of parts of the human body. Technical report, International Organization for Standardization.

ISO/TS 15066 (2016). Robots and robotic devices - collaborative robots. Technical report, International Organization for Standardization.

Lippi, M. and Marino, A. (2018). Safety in human-multi robot collaborative scenarios: a trajectory scaling approach. IFAC-PapersOnLine, 51(22):190-196.

Ross, T. J. (2010). Fuzzy Logic with Engineering Applications. John Wiley \& Sons, Ltd.

Rusu, R. B. and Cousins, S. (2011). 3d is here: Point cloud library (PCL). In IEEE International Conference on Robotics and Automation. IEEE.

The LABOR project (2019). https://www.labor-project.eu/.

Torr, P. and Murray, D. (1997). The development and comparison of robust methods for estimating the fundamental matrix. International Journal of Computer $\mathrm{Vi}$ sion, 24(3):271-300.

Zhang, P., Jin, P., Du, G., and Liu, X. (2016). Ensuring safety in human-robot coexisting environment based on two-level protection. Industrial Robot: An International Journal, 43(3):264-273. 